\title{
Parteras tradicionales y su relación con las instituciones de salud. Entre la resistencia y la subordinación
}

\author{
SILVIA JIMÉNEZ ${ }^{(1)}$, BLANCA PELCASTRE(2) y JUAN GUILLERMO FIGUEROA ${ }^{(3)}$
}

\section{RESUMEN}

El objetivo es conocer cómo las parteras tradicionales construyen su saber en salud materna y cómo se relacionan con las instituciones de salud. ${ }^{\text {(a) }}$

Se realizó un estudio cualitativo en el 2005, se entrevistaron en sus domicilios a ocho parteras tradicionales, se observaron tres consultas prenatales y dos cursos de capacitación para parteras en Morelos, México. Las guías de entrevista y observación comprendieron temáticas sobre iniciación como parteras, relación con las instituciones de salud, así como utilización de prácticas tradicionales y biomédicas.

Las parteras tradicionales combinan conocimientos tradicionales y biomédicos. Este último por influencia de las instituciones de salud a través de cursos de capacitación. Estas mujeres se han visto expuestas al rechazo médico, lo que ha ocasionado ciertos conflictos en la relación. La situación las ha obligado a ocultar que son parteras o no acompañar a sus pacientes a la atención médica con el fin de que sus pacientes accedan a la atención médica. Como forma de resistencia ante la crítica médica llevan a cabo prácticas tradicionales que consideran benéficas para las mujeres.

El proceso de medicalización que prevalece en las instituciones de salud ha repercutido de forma importante en la partería tradicional. Las parteras se han expuesto a la exclusión y deslegitimización de sus prácticas tradicionales; sin embargo, han adquirido conocimientos biomédicos para mejorar la calidad de su atención.

Palabras clave: parteras tradicionales, modelo biomédico, medicina tradicional, resistencia, subordinación.

\section{ABSTRACT}

TRADITIONAL MIDWIVES AND THEIR RELATION WITH HEALTH INSTITUTIONS. THE FIGHT BETWEEN RESISTANCE AND SUBORDINATION.

Purpose: To know how traditional midwives build their maternal health knowledge and how they relate with health institutions. Methods: A qualitative study was carried out in 2005. In such study eight traditional midwives were interviewed at their homes, and three antenatal consultations and two training courses for midwives were assessed in Morelos, Mexico. Interview and observation

(1) Instituto Mexicano del Seguro Social. Unidad de Salud Pública. Coordinación de Vigilancia Epidemiológica y Apoyo en Contingencias. Mier y Pesado 120. Col. Del Valle. México, D.F. C.P. 03100 silvia.jimenez@imss.gob.mx

(2) Instituto Nacional de Salud Pública. México.

(3) El Colegio de México. México.

(a) Las parteras tradicionales en México guardan ciertas analogías con las matronas en el contexto de Chile, debido al tipo de trabajo que realizan al acompañar a las mujeres durante el embarazo y en el parto. 
guidelines included topics such as the beginnings as a midwife, the relation with health institutions as well as the observance of traditional and biomedical practices. Results: Traditional midwives combine traditional and biomedical knowledge. The latter is a result of the influence of health institutions carried out through training courses. These women have been exposed to the rejection attitude of physicians that has generated relationship conflicts, the situation leading them to hide their midwife nature or not to escort their patients to medical consultation in order to preserve their patient's physician medical care. As a means of resistance before the medical criticism, they carry out traditional practices they consider beneficial to women. Conclusions: The medicalization process prevailing in health institutions has had a significant impact on traditional midwifery. Midwives have been exposed to exclusion and illegitimation of their traditional practices, however, they have acquired biomedical knowledge to improve the quality of the care provided.

Keywords: Traditional midwives. Biomedical model. Traditional medicine. Resistance. Subordination.

\section{INTRODUCCIÓN}

Las parteras tradicionales son personas que surgen de su propia comunidad y que por tradición, convivencia y vocación se dedican a atender partos y algunos problemas de salud, de acuerdo a los hábitos y costumbres de la región donde prestan sus servicios. Esto las convierte en líderes naturales aceptadas y reconocidas por la comunidad. En México, debido al acceso desigual a la asistencia médica y/o por las preferencias culturales de la población, siguen siendo una importante alternativa para miles de mujeres ${ }^{1,2}$. $\mathrm{Su}$ actividad principal que durante siglos había sido la atención del parto, paulatinamente ha disminuido. En 1981, atendieron en las comunidades rurales de México -menos de 2.500 habitantes- el $54.4 \%$ de los partos; $30 \%$ en $1997 ; 17 \%$ en el 2000 ; y $13.7 \%$ en $2003^{3-6}$. En este descenso ha influido de manera importante la medicalización del embarazo y del parto.

En algunos lugares de México, las parteras han tenido un importante papel en dicho proceso de medicalización, ya que muchas llegan a ser el primer agente de salud que visitan las mujeres ante un problema de salud. Como primer contacto, han facilitado el camino para que las acepten y busquen atención médica formal. Sin embargo, los distintos momentos en los cuales se establece una relación entre parteras e instituciones de salud han tenido repercusiones en el proceso de la atención médica institucional y en la forma en que las parteras prestan sus servicios, estableciéndose así un efecto en ambos sentidos. En virtud de ello, el objetivo de esta investigación fue conocer y comprender cómo las parteras construyen su saber en el ámbito de la salud materna y, en este proceso de vinculación institucional, cómo han establecido su relación con el personal de salud y con las mujeres que atienden.

\section{MATERIAL Y MÉTODO}

A través de la metodología cualitativa, se llevaron a cabo ocho entrevistas semiestructuradas a parteras tradicionales capacitadas por el Instituto Mexicano del Seguro Social (IMSS), se observaron tres consultas prenatales proporcionadas por parteras en sus domicilios y dos reuniones de capacitación en el Hospital General Regional N ${ }^{\mathrm{o}} 1$ del Instituto Mexicano del Seguro Social (institución que brinda servicios de salud a personas que trabajan en instituciones del sector privado), en Cuernavaca, Morelos.

Para seleccionar a las participantes, se utilizó la base de datos del Registro Individual de Parteras 2003 del IMSS de la delegación Morelos, que contiene información sobre la ubicación de las parteras, así como algunas características de 
su trabajo. Se seleccionó a aquellas que residían en Cuernavaca y Temixco y que, además, proporcionaban en promedio diez consultas prenatales al mes; su participación fue voluntaria y confidencial $^{(\mathrm{b})}$.

La muestra de parteras no fue construida con el fin de que tuviera representatividad estadística y desde ahí generalizar los resultados como tradicionalmente se busca en las aproximaciones cuantitativas, sino que buscamos heterogeneidad en las entrevistas hasta alcanzar el principio de saturación teórica, es decir, cuando ya no obtuvimos más información heterogénea de la que ya habíamos recopilado. Esto no le resta relevancia ni validez al estudio, ya que dicha diversidad pretende profundizar en las diversas modalidades de parteras que existen en México y así enriquecer el entorno desde donde se estudian. Esta aproximación metodológica privilegia la búsqueda de una población que diversifique las observaciones y desde ahí, más que medir la incidencia de las mismas, se trata de interpretar las diferentes experiencias observadas ${ }^{7-12}$. En este sentido, los resultados son válidos en el contexto donde laboran las parteras tradicionales y que han sido capacitadas por instituciones públicas de salud, pero - a la vez - en "contextos similares", interpretado esto en función del conocimiento que tengan las personas que lo lean respecto de las poblaciones con las que se pueden llegar a comparar estos resultados ${ }^{13}$.

El trabajo de campo se realizó de febrero a septiembre del 2005, las entrevistas se grabaron y se transcribieron literalmente. Para el análisis, se utilizó el programa de cómputo Atlas-ti (V. 4.2). Durante la revisión del material estuvimos constantemente diseñando y rediseñando el análisis de la información para darle sentido a lo que encontramos y no caer en ambigüedades; tratamos de conceptualizar, categorizar y codificar de acuerdo a los esquemas encontrados. Se identificó un listado de categorías analíticas, las cuales fueron: a) relación con las instituciones de salud; b) prácticas antes y después de la capacitación; $y, c)$ imposición y resistencia al modelo médico hegemónico. Posteriormente se realizó el proceso de codificación de cada una de las entrevistas.

\section{RESULTADOS}

\section{Características socioeconómicas}

Las ocho parteras entrevistadas habían tenido al menos un hijo; tres estaban casadas o vivían en unión libre, tres se habían separado de sus parejas y dos eran viudas; su edad fluctuó entre 45 y 88 años. Se identificaron niveles de escolaridad altos, una partera estudió, sin concluir, una licenciatura en administración de empresas, dos terminaron la secundaria y dos la primaria; las otras tres no culminaron sus estudios de primaria o no asistieron a la escuela.

\section{Relación con las instituciones de salud y el inicio de su medicalización}

Las parteras iniciaron su relación con las instituciones de salud hace más de una década. Fueron capacitadas con el fin de que aprendieran a identificar riesgos en las embarazadas y las derivaran a un servicio médico. Esta situación que tiene como intención la disminución de las muertes maternas y neonatales, ha sido difícil para las parteras ya que al embarazo y al parto los habían considerado como un evento natural y familiar. Sin embargo, ahora han modificado esta creencia y han aceptado que no pueden atender a las embarazadas con complicaciones, sino deben hacerlo los médicos, iniciándose en ellas un proceso de subordinación al modelo biomédico. El siguiente testimonio ilustra lo anterior.

(b) Las parteras firmaron o pusieron su huella en una hoja de consentimiento informado donde se especificaban los objetivos del estudio, la confidencialidad de su participación y que la entrevista sería grabada, además se les solicitó su autorización para que sus testimonios fueran utilizados en una publicación. 
- ¿Cambió su forma de atender a las mujeres cuando recibió su primer curso de capacitación? - Hemos mejorado muchas cosas que no sabía. Por ejemplo a tomar presión ¿yo cuándo? Ya nomás me guiaba yo por lo que sentía -tiene su presión baja o su presión alta-. Ahora no, ya se las tomo. A partir de que empecé a ir a los cursos. Sí, nos han servido mucho, porque ya ahí nos dicen que presión alta, que no, que presión baja, que no, que personas jóvenes, no, que personas grandes, no. Tengo más capacitación gracias a las instituciones (partera: 290-328, 56 años).

Las parteras han aprendido a identificar problemas biológicos en las embarazadas, lo que sin duda contribuye a la disminución de complicaciones y/o muertes maternas y perinatales. Sin embargo, las ha limitado en su actuación, situación que ha servido para marcar las diferencias entre su saber tradicional y el saber biomédico, dando pie a conflictos entre estos actores sociales que tienen una diferente concepción del mundo, como se analiza más adelante.

\section{Combinación de la medicina hegemónica y la tradicional}

Las parteras han adquirido conocimientos tanto biomédicos como tradicionales, lo que ha provocado una transformación en su quehacer. A través de la biomedicina han aprendido a inyectar, poner sueros, medir la presión arterial, hacer tactos vaginales, recetar medicamentos alópatas, prescribir anticonceptivos, insertar dispositivos intrauterinos e incluso tomar pa- panicolaou. Para la identificación de riesgos en las embarazadas, realizan diferentes acciones. Así lo observamos en tres consultas prenatales: interrogan a las mujeres sobre sus antecedentes obstétricos y síntomas durante el embarazo, además preguntan sobre los meses de embarazo e indagan sobre la dieta. También miden algunos signos vitales como la presión arterial, la temperatura corporal, y para identificar la evolución del bebé, miden el vientre de las madres, las pesan, escuchan el corazón del bebé y miden su frecuencia cardíaca.

En su formación como parteras tradicionales, han desarrollado habilidades manuales para identificar la posición de un bebé. Así cuando identifican que un bebé no está en posición correcta, lo tratan de acomodar, utilizando prácticas tradicionales como "sobar", "mantear" o "componer"(c). Observamos en una consulta, que una partera atendió a una joven de 16 años con ocho meses de embarazo, le palpó el vientre y percibió que tenía "encajado" al niño del lado derecho por lo que procedió a acomodarlo, así que pidió a la mujer que se colocara sobre una silla acondicionada con una tabla; la embarazada se colocó sobre ella con la cabeza hacia abajo. La partera fue palpando el vientre de la mujer suavemente; después de realizar las maniobras, desde la perspectiva de la partera, ya había acomodado al bebé. Otra práctica tradicional que utilizan es el baño de temascal; pudimos apreciar que dos parteras cuentan con este servicio, quienes por costumbre, bañan a las mujeres después de ocurrido el parto, para lo cual, utilizan ciertas

(c) La partera palpa el vientre para localizar la cabeza del niño y determina su posición, si detecta que no es la correcta, con movimientos precisos y firmes y ayudada con ambas manos, trata de desplazarlo mediante un movimiento giratorio en dirección de la posición adecuada. La sobada al final del embarazo permite a la partera establecer la fecha probable del parto y detectar la posición fetal ${ }^{14}$.

(d) El baño de temazcal es conocido también como "la casa del sudor", su significado literal en Náhuatl es "la casa del baño"; tiene forma de iglú y representa al vientre materno de la madre tierra y de alguna manera lo que se experimenta es un renacimiento. La puerta representa al útero de donde salimos de nuestra madre y el entrar de nuevo es un acto sagrado de regresar por donde venimos al mundo, el interior oscuro, caliente y húmedo nos contiene y protege como el vientre de nuestra madre nos contuvo y nos protegió. Las parteras lo utilizan después del alumbramiento para sacar la frialdad del cuerpo y fortalecer los huesos de la cadera de la madre ${ }^{15}$. 
hierbas medicinales ${ }^{(\mathrm{d})}$.

La mezcla de prácticas tradicionales y biomédicas les ha permitido ampliar su gama de posibilidades para la atención que ofrecen en sus comunidades; sin embargo, al no abandonar la medicina tradicional las ha llevado a enfrentarse a ciertos conflictos con las instituciones de salud.

\section{Rechazo a las prácticas tradicionales}

El saber popular de las parteras se ha caracterizado por un proceso constante de modificación, donde se sintetizan concepciones y prácticas derivadas de diferentes saberes, incluido el biomédico.

La forma de atención tradicional es por lo general negada, ignorada $\mathrm{y} / \mathrm{o}$ marginada por parte de la biomedicina, pese a que la atención tradicional es aún en algunos lugares frecuentemente utilizada por diferentes sectores de la población ${ }^{16}$. Las parteras son uno de los diversos terapeutas que se apoyan en la medicina tradicional; $y$ pese a que identifican a la medicina institucional como la forma más correcta y eficaz de atención, también consideran benéficas ciertas prácticas tradicionales y optan por no abandonarlas, estableciéndose entonces un mecanismo de oposición o resistencia a las representaciones y prácticas médicas hegemónicas ${ }^{17-19}$. El siguiente testimonio da cuenta de esta situación:

- ¿Por qué los médicos no están de acuerdo con las sobadas?

- Por lo mismo, que dicen pasa a amolar más al bebé, nada más así. No es así, porque muchas veces acomodando al bebé llega a ser normal, y cuando el bebé está atravesado, ellos por ejemplo, no, no acomodan. Ellos dicen: está atravesado, va a ser cesárea. Y no mueven un dedo. Y nosotras no, nosotras lo intentamos a que el bebé se acomode (partera 5: 622-630, 56 años).

\section{EI dilema de derivar}

Las parteras tradicionales han contribuido a facilitar la accesibilidad para la utilización de los servicios médicos ${ }^{1-2}$. Una de las muchas formas que han ideado para que las mujeres acepten acudir a los servicios médicos es acompañándolas.
Sin embargo, este hecho les ha ocasionado experimentar diversas formas de maltrato por parte de los médicos, como se ilustra en el siguiente testimonio:

"Una vez tuve una mujer que se le rompieron las membranas y tenía cinco de dilatación, y el bebé estaba grande, era primeriza; y la llevo al hospital, yo le estoy tomando la presión, si es cada media hora, cada hora, le estoy tomando la presión, le estoy tomando el pulso, todo, porque lo he aprendido así, gracias a Dios a las instrucciones de tener todo eso que nos puede ayudar a mucho. Entonces, llevo anotado todo en el expediente y llego a emergencias, paso con la mujer y le digo al médico: viene con presión. Con perdón de usted la palabra vulgar que dicen, no me peló."

\section{-¿Y sabía que usted era partera?}

-Sabía que yo era partera. Pero así, muy indiferente, como quien dice -está loca, qué le voy hacer caso-. Entonces, no se vale, no se vale, porque nosotras ya estamos capacitadas y ya sabemos que, si estamos llevando a una paciente es para prevenir que suceda algo, [...] cuando vi que ya que no avanzaba, de 5 de dilatación, y ya tenía 5 horas con las membranas rotas, dije no, me la llevo ¿yo por qué me voy arriesgar? Entonces que le hablo a un doctor [médico particular que la apoya en casos de emergencia] y le dije -isabes qué? prepara el quirófano, ahorita te llevo a una paciente. Bajo mi responsabilidad la fui a sacar del hospital [...]. (partera 7: 851-903, 49 años).

Este testimonio muestra que las parteras se han sensibilizado sobre la importancia de derivar a un servicio médico cuando identifican riesgos. Sin embargo, no siempre cuentan con el apoyo de las instituciones de salud. Por otro lado, algunas parteras han encontrado una red de apoyo en los médicos particulares, esta situación refleja la ambivalencia entre tensiones evidentes, pero también posibles momentos de complementariedad entre parteras y médicos.

Al respecto, las parteras han optado por no acompañar a sus pacientes o si lo hacen no se presentan como tales ante las instituciones de 
salud; sin embargo, la crítica médica sigue dándose. Así lo refleja el siguiente testimonio:

- [...] las acompaño, y yo allá voy a decir nomás que es mi vecina, -tú vas a decir tus datos, yo te acompaño-. Y luego ya no dejan entrar a uno, nomás la pura muchacha [...]

- ¿Cómo se siente de no poder decir que es partera?

-Porque ahí nos regañan o les están reprochando que por qué se va a quedar conmigo. Yo les platico antes: mira, te voy a llevar, pero tú no digas que yo te mando, que yo te llevo, que yo soy partera. Bueno, yo sí te llevo, pero la que vas a dar tu nombre eres tú, y tu vas a decir que pues vas por primera vez. Porque si yo le estuve llevando el control, ahí no las quieren recibir porque no fueron antes, y es que se enojan. (Partera 1: 490508, 64 años).

Los distintos momentos en que las parteras se ven expuestas a la crítica médica muestra cómo son deslegitimadas social y técnicamente, lo que atenta contra su dignidad personal ${ }^{(\mathrm{e})}$, y las obliga a dejar de acompañar a las mujeres o silenciar su profesión. Estos comportamientos pueden ocasionar un daño a la salud de las mujeres y sus hijos, ya que está latente el retraso en la derivación y en la atención médica. Sería importante conocer la oportunidad con que las parteras están enviando a las mujeres a los servicios médicos, pero también, conocer la oportunidad de la respuesta institucional a las derivaciones hechas por las parteras.

Otra forma en que las parteras han percibido el rechazo médico es porque han percibido que los galenos o médicos les tienen "celo profesional", a continuación un testimonio que ilustra este aspecto:
- ¿Por qué cree que los médicos son así con ustedes?

-Como que hay un celo profesional, como que dicen -éstas ni han estudiado, ni han gastado, ni han pasado noches enteras quemándose las pestañas, y ganan más que nosotros porque atienden más partos que nosotros-. Pero ¿por qué no se han puesto a pensar? Bueno ¿por qué estas mujeres buscan más a las parteras? Buscar el por qué ¿no? Y siempre decimos -es la calidez, es la paciencia- ¿Por qué no ponen eso ellos en su práctica la paciencia y la calidez? (partera 8 : 1020-1030, 45 años).

Llama la atención la forma en que la partera percibe el rechazo porque no estudió en una universidad, es decir, no dispone del conocimiento oficial. Sin embargo, ella considera que las mujeres buscan sus servicios no sólo para la vigilancia del embarazo o la atención del parto, sino porque les proporciona un trato cálido y humanitario que desde su perspectiva no ofrecen los médicos. El saber escuchar es una práctica cotidiana de las parteras, lo que muestra la empatía que tienen con sus pacientes, contrario a lo que desde su perspectiva practican los médicos.

Como se ha visto, los distintos momentos que el personal de salud institucional, especialmente los médicos tienen para deslegitimar el saber de las parteras tradicionales, también sirve para legitimar la hegemonía de la biomedicina y advertir tanto a las parteras como a sus pacientes que la mejor alternativa es la atención médica.

\section{DISCUSIÓN}

El proceso de medicalización del modelo médico que prevalece hoy en día en las instituciones de salud, ha repercutido de forma importante en

(e) La dignidad es un atributo tanto privado como público. Alguien puede sentir que otro lo ha ultrajado aunque no haya testigos. Por otro lado, es bastante claro que, cuando se realiza en público, el ultraje se agrava enormemente. Un insulto, una mirada de desprecio, una humillación física, un ataque a la calidad y a la posición de la persona, una grosería, son casi siempre mucho más injuriosos cuando ocurren en presencia de testigos ${ }^{20}$. 
la práctica de las parteras tradicionales quienes se han vinculado con estas instituciones, estableciéndose entre ellas relaciones de poder-subordinación ${ }^{21}$. Como producto de estas relaciones, las parteras empiezan a perder cierta libertad en el ejercicio de su profesión, subordinándose a un modelo predominante, que de acuerdo a Menén$\operatorname{dez}^{22-24}$ y Tetelboin ${ }^{25}$ trata de excluir y deslegitimar prácticas de la medicina tradicional.

A partir de estas relaciones de poder, que desde el punto de vista del paradigma estratégico ${ }^{(\mathrm{f})}$ se establecen, las parteras han desarrollado prácticas de resistencia que funcionan como barreras para evitar el conflicto. Desde un punto de vista muy sintético y operativo, las prácticas de resistencia pueden definirse como todas aquellas alternativas frente a la cultura y comunicación hegemónicas ${ }^{21,26-27}$. En este estudio observamos que una de estas prácticas que llevan a cabo las parteras es ocultar su profesión cuando se encuentran en los espacios médicos con el fin de evitar el rechazo médico. Otra forma de resistir, es la postura escéptica que mantienen frente a la medicina y al discurso institucional, conduciéndose con cautela, ya que en esas instituciones no siempre se reconoce la importancia técnica y cultural de su trabajo.

El dominio del modelo médico que prevalece para la atención del parto, en virtud de su carácter hegemónico, tiende a desarticular los lazos sociales e ideológicos que las parteras han construido durante generaciones con las mujeres de sus comunidades, e intenta sustituir sus prácticas por otras que se consideran adecuadas. En este estudio observamos que las prácticas de resistencia de las parteras no siempre son voluntarias, sino que las personas de sus comunidades también ejercen sobre ellas una presión que las hace actuar en consecuencia. En este sentido, la capacitación que reciben por parte de las instituciones oficiales de salud puede significar la oportunidad de legitimarse frente a la práctica médica, de ser un medio a través del cual obtengan el respeto del que carecen ante la comunidad médica y que les ha costado desprestigio en sus comunidades de origen.

De esta forma, las parteras tradicionales se han vinculado al sistema médico formal y se han conducido entre la resistencia y la subordinación para que su práctica, ante todo, sobreviva. Son muchas todavía las acciones que deben llevarse a cabo para lograr un verdadero modelo de capacitación en el que prevalezca el intercambio por encima de la imposición o sustitución de prácticas. Toda una reflexión a partir de la perspectiva y la experiencia del personal médico queda pendiente.

\section{REFERENCIAS}

1. LUENGAS AGUIRRE, MIF. El saber y el quehacer de las parteras en el medio rural mexicano. Tesis de Maestría, Facultad de Ciencias Políticas y Sociales. UNAM; 1994.

2. JIMÉNEZ GARCÍA SE, Loggia Gago, SM, Taracena, $\mathrm{R}$, Magis, $\mathrm{C}$ y Gayet $\mathrm{C}$. El trabajo de las parteras sobre VIH/SIDA y otras Infecciones de Transmisión Sexual. Ángulos del SIDA; 2004.

3. INSTITUTO MEXICANO DEL SEGURO SOCIAL. Encuesta Rural de Planificación Familiar. Resultados Nacionales; 1981.

4. CONSEJO NACIONAL DE POBLACIÓN. Encuesta Nacional de Planificación Familiar, 1995 (Estimaciones propias utilizando la base de datos de la encuesta).

5. SECRETARÍA DE SALUD. Encuesta Nacional de Salud, 2000 (Estimaciones propias utilizando la base de datos de la encuesta).

6. SECRETARÍA DE SALUD. Encuesta de Salud Reproductiva, 2003 (Estimaciones propias utilizando la base de datos de la encuesta).

7. GLASSER BG, STRAUSS A. The Discovery of Grounded Theory: Strategies for Qualitative Research. Chicago; Aldine, 1967.

8. CASTRO, R. En busca del significado: supuestos, alcances y limitaciones del análisis cualitativo. En: Szasz, I. y Lerner, S. Para comprender la subjetividad. Investigación cualitativa en salud reproductiva y sexualidad. El Colegio de

(f) El paradigma estratégico concibe el poder como una relación en contraste con el paradigma jurídico, que lo considera una propiedad o sustancia ${ }^{21}$. 
México, 1999:57-88.

9. CASTRO, R. Y BRONFMAN M. Problemas no resueltos en la integración de métodos cualitativos y cuantitativos en la investigación social en salud. En: Bronfman M. y Castro R. (coordinadores) Salud, cambio social y política. Perspectivas desde América Latina. EDAMEX e Instituto Nacional de Salud Pública. México, 1999: 4964.

10. CORTÉS, F. Algunos aspectos de la controversia entre la investigación cualitativa y la investigación cuantitativa. En: Cortés F., Escobar A. y González de la Rocha M. Método científico y política social. A propósito de las evaluaciones cualitativas de programas sociales. El Colegio de México. 2008: 27-58.

11. FLYVBJERG, BENT. The power of example (Cases and 'casing'). En: Making Social Science Matter. Cambridge University Press, 2006: 7687.

12. GUADARRAMA, R. Las paradojas actuales de la investigación cualitativa en ciencias sociales. En: Canales A. y Lerner S. (coordinadores). Desafíos teóricos y metodológicos en los estudios de población en el inicio del milenio. El Colegio de México, Universidad de Guadalajara y Sociedad Mexicana de Demografía. México, 2003: 162-176.

13. TARRÉS, M. L. Lo cualitativo como tradición. En: Tarrés, M. (coordinadora). Observar, escuchar y comprender. Sobre la tradición cualitativa en la investigación social. El Colegio de México, FLACSO y Editorial Porrúa, 2008: 35-60.

14. GUBA, E. Y LINCOLN Y. S. Paradigmas en competencia en la investigación cualitativa. En: Denman, C y Haro J.A. (compiladores). Por los rincones. Antología de métodos cualitativos en la investigación social. El Colegio de Sonora, Hermosillo, México, 2000: 113-145.

15. HERAS, A. Y ADAYA, Y. Plantas medicinales usadas en el baño de Temazcal de Atlahuatla, Estado de México. Tlahui-Medic, 2004.

16. GÜÉMEZ PINEDA, M. La concepción del cuerpo humano, la maternidad y el dolor entre mujeres mayas yucatecas. Universidad Autónoma de Yu- catán. Rev Mesoamérica, 2000; 21(39):305-33.

17. MENÉNDEZ, E. Modelos de atención de los padecimientos: de exclusiones teóricas y articulaciones prácticas. En: Ciência \& Saûde Colectiva, Río de Janeiro. 2003.

18. MENÉNDEZ, E. La enfermedad y la curación. ¿Qué es medicina tradicional? ALTERIDADES. 1994, 4 (7):71-83.

19. MENÉNDEZ, E. L. La parte negada de la cultura. Relativismo, diferencias y racismo. Edición Bellaterra; 2002.

20. SCOTT, J. C. Los dominados y el arte de la resistencia. Discursos ocultos. Colección Problemas de México, Ediciones ERA; 2004.

21. IBÁÑEZ, T. Poder y Libertad. Estudio sobre la naturaleza, las modalidades y los mecanismos de las relaciones de poder. Biblioteca HORA. Barcelona; 1982.

22. MENÉNDEZ SPINA EL Y DI PARDO RB. De algunos alcoholismos y algunos saberes. Atención primaria y proceso de alcoholización. Ciesas, Colección Miguel Othón Mendizábal. México; 1996.

23. MENÉNDEZ, E. Saber local y toma de decisiones. En: Haro A y De Keijzer B. Coords. Participación comunitaria en salud: evaluación de experiencias y tareas para el futuro. El Colegio de Sonora, México, 1998.

24. MENÉNDEZ, E. Intencionalidad, experiencia y función: la articulación de los saberes médicos. CIESAS-SPAM, México; 2004.

25. TETELBOIN, C. Problemas en la conceptualización de la práctica médica. En: Antrop. Biol. 1984; VIII: 487-510.

26. FOUCAULT, M. Microfísica del Poder. Madrid, España. Las Ediciones de la Piqueta; 1992.

27. FOUCAULT, M. El sujeto y el poder. Chicago University Press, 1983 (traducción de Santiago Carassale y Angélica Vitale).

Recepción: 14 de octubre de 2008 Aprobación: 12 de diciembre de 2008

Usted puede comentar éste y otros artículos publicados en la Revista Chilena de Salud Pública, enviando un correo electrónico a revistasp@med.uchile.cl 